IRSTI 27.35.51

\title{
Growth rates of space-charge waves in a Lorentzian plasma waveguide
}

\author{
Myoung-Jae Lee ${ }^{1}$ and Young-Dae Jung ${ }^{2 *}$ \\ ${ }^{1}$ Department of Physics, Hanyang University, Wangsimni-ro, Seongdong-gu, \\ Seoul 04763, South Korea \\ ${ }^{2}$ Department of Applied Physics and Department of Bionanotechnology, \\ Hanyang University, Ansan, Kyunggi-Do 15588, South Korea \\ *e-mail:ydjung@hanyang.ac.kr
}

\begin{abstract}
The non-thermal effects on the growth rate of the space charge wave are investigated in a Lorentzian dusty plasma-filled waveguide by employing the normal mode analysis and the method of separation of variables. The growth rate and the real frequency of the space charge wave are obtained as functions of the harmonic roots of the Bessel function and the spectral index of the Lorentzian plasma. It is shown that the non-thermal effect suppresses the real frequency of the space-charge wave. In addition, the scaled growth rate decreases with an increase of the spectral index. Hence, we have found that the growth rates in non-thermal plasmas are always smaller than those in thermal plasmas. It was shown that the influence of non-thermal character plays a crucial role on the space-charge wave due to the coupling interaction of the streaming ions with non-thermal electrons in a Lorentzian dusty plasma waveguide. These results would provide useful information on the nonthermal effects on the space-charge wave in non-Maxwellian multi-component plasma waveguides.
\end{abstract}

Key words: Lorentzian waveguide, space-charge wave, Bessel function, spectral index, Lorentzian plasma. PACS numbers: 52.35.Fp, 52.35.-g, 52.20.-j

\section{Introduction}

The plasma instability has been extensively explored since this phenomenon is one of the most interesting wave-particle interactions as well as the effective heating mechanisms in various plasmas [1-3]. The characteristics of surface plasma waves have been extensively explored in numerous bounded plasmas since the dispersion relations have provided detailed information on the plasma parameters and geometrical effects of the plasma system [4-8]. There has been a considerable interest in dynamics and structures of complex plasmas containing the component of highly charged dust grains including the strong collective interactions [9-12]. Recently, the Lorentzian distribution or the kappa distribution has been of a great interest since it has been shown that the Lorentzian distribution is considerably useful for exploring various physical properties of non-thermal dusty plasmas illustrating high-energy tails which strongly deviate from the standard Maxwellian distribution due to the coupling between plasma particles and external fields [13-15]. Then, it would be then expected that the physical properties of the beam-instability of space-charge waves in a Lorentzian plasma waveguide are quite different from those in bulk plasmas due to the influence of non-thermal properties of the Lorentzian plasma and the harmonic-modes of the Bessel function for the cylindrical geometry. Hence, in this paper we investigate the non-thermal effects on the spacecharge wave in a Lorentzian dusty plasma waveguide including streaming ions since it would be expected that the coupling between the resonant wave-particle interaction, non-thermal and geometrical characters of the Lorentzian plasma provides the physical understanding of the coupling mechanism and useful information on the dispersion properties of the complex plasma system. We then obtain the dispersion relation and the instability mode for the space-charge wave in a Lorentzian dusty plasma waveguide by employing the normal mode analysis and the method of separation of variables.

\section{Numerical Simulations}

It has been shown that superthermal particles would be generated by the interaction between the equilibrium velocity distributions with the strong external fields. The plasma containing the non- 
thermal tail for the electron distribution would be represented by the generalized Lorentzian velocity distribution $f_{k}\left(v_{e}\right)$ represented by the following form [13]:

$$
f_{\kappa}\left(v_{e}\right) d v_{e}=4 \pi\left(\frac{m_{e}}{2 \pi \kappa E_{\kappa e}}\right)^{3 / 2} \frac{\Gamma(\kappa+1)}{\Gamma(\kappa-1 / 2)}\left(1+\frac{m_{e} v_{e}^{2}}{2 \kappa E_{\kappa e}}\right)^{-(\kappa+1)} v_{e}^{2} d v_{e}
$$

where $k(>3 / 2)$ is the spectral index, $v_{e}$ is the velocity of the electron, $m_{e}$ is the mass of the electron, $\quad E_{\kappa e}\left[=k_{B} T_{e}(\kappa-3 / 2) / \kappa\right] \quad$ is the characteristic electron energy in the Lorentzian plasma, $\Gamma$ stands for the Gamma function, $k_{B}$ is the Boltzmann constant, and $T_{e}$ is the electron temperature. For the frequency domain $\omega_{p d}<<\omega<\omega_{p i}$, the plasma dielectric function [12] of the Lorentzian dusty plasma composed of the non-thermal Lorentzian electrons, streaming ions, and stationary dusty grains would be represented by

$\varepsilon(\omega, k) \cong 1+\frac{1}{k^{2} \lambda_{\kappa e}^{2}}-\frac{\omega_{p i}^{2}}{\left(\omega-\mathbf{k} \cdot \mathbf{u}_{i}\right)^{2}}-\frac{\omega_{p d}^{2}}{\omega^{2}}$,

$\omega$ is the frequency, $k$ is the wave number, $\lambda_{\kappa \alpha}\left(\equiv \mu_{\kappa} \lambda_{D \alpha}\right)$ is the effective Debye length in the Lorentzian plasma, $\mu_{\kappa} \equiv[(2 \kappa-3) /(2 \kappa-1)]^{1 / 2}$, $\lambda_{D \alpha}$ is the standard Debye length of species $\alpha$ in Maxwellian plasmas, since the Doppler-shifted frequency would be given by $\omega-\mathbf{k} \cdot \mathbf{u}_{i}$ for the ion streaming with the velocity $\mathbf{u}_{i}$. In unmagnetized complex plasmas, the continuity and momentum equations for the species $\alpha$ of plasma particle, i.e., $\alpha=e$ (electron), $i$ (ion), $d$ (dust), and Poisson's equation are, respectively, given by

$$
\begin{gathered}
\frac{\partial n_{\alpha}}{\partial t}+\nabla \cdot\left(n_{\alpha} \mathbf{v}_{\alpha}\right)=0 \\
m_{\alpha} n_{\alpha}\left(\frac{\partial \mathbf{v}_{\alpha}}{\partial t}+\mathbf{v}_{\alpha} \cdot \nabla \mathbf{v}_{\alpha}\right)=-\nabla P_{\alpha}-q_{\alpha} n_{\alpha} \nabla \phi \\
\nabla^{2} \phi=-4 \pi \sum_{\alpha=e, i} q_{\alpha} n_{\alpha}
\end{gathered}
$$

where $n_{\alpha}\left(=n_{\alpha 0}+n_{\alpha 1}\right), \mathbf{v}_{\alpha}\left(=\mathbf{v}_{\alpha 0}+\mathbf{v}_{\alpha 1}\right), m_{\alpha}$, $P_{\alpha}, q_{\alpha}$, and $\phi$ are the number density, velocity, mass, pressure, charge of the electron, and electric potential, respectively. In order to describe the cylindrical waveguide filled with complex plasma composed of the non-thermal Lorentzian electrons, streaming ions, and stationary dusty grains, we assume that the plasma waveguide has the sufficiently long column along the $z$-axis with the radius $R$. In this cylindrical system $(\rho, \theta, z)$, the all perturbation quantities $n_{\alpha 1}(\mathbf{r}), \mathbf{v}_{\alpha}(\mathbf{r})$, and $\phi(\mathbf{r})$ would be expressed by the wave-like oscillating dependence [16] with the separation of variables such as

$$
\left[\begin{array}{c}
n_{\alpha 1}(\mathbf{r}) \\
\mathbf{v}_{\alpha 1}(\mathbf{r}) \\
\phi(\mathbf{r})
\end{array}\right]=\left[\begin{array}{c}
\bar{n}_{\alpha 1}(\rho) \\
\overline{\mathbf{v}}_{\alpha 1}(\rho) \\
\bar{\phi}(\rho)
\end{array}\right] \exp (-i \omega t) \exp [i(k z-\mu \theta)]
$$

where $\quad \bar{n}_{\alpha 1}(\rho), \quad \overline{\mathbf{v}}_{\alpha 1}(\rho)$, and $\bar{\phi}(\rho)$ are the perturbation quantities in the transverse plane, $\mu$ is the azimuthal constant, and $k_{z}\left[=\left(k^{2}-k_{\perp}^{2}\right)^{1 / 2}\right]$ is the wave number along the axial $z$-direction. For the azimuthally symmetric case in cylindrical coordinates: $\partial / \partial \theta=0$ [17], i.e., $\mu=0$, the differential equation for the potential field $\bar{\phi}(\rho)$ becomes 


$$
\frac{d^{2} \bar{\phi}(\rho)}{d \rho^{2}}+\frac{1}{\rho} \frac{d \bar{\phi}(\rho)}{d \rho}+k_{z}^{2}\left[-1-\frac{1}{k_{z}^{2} \lambda_{\kappa e}^{2}}+\frac{\omega_{p i}^{2}}{\left(\omega-k_{z} u_{i}\right)^{2}}+\frac{\omega_{p d}^{2}}{\omega^{2}}\right] \bar{\phi}(\rho)=0 .
$$

The general solution of Eq. (9) is then represented by $\bar{\phi}(\rho)=a_{1} J_{0}(\xi \rho)+a_{2} N_{0}(\xi \rho)$, where $\alpha_{1}$ and $\alpha_{2}$ are constant coefficients of the solution, $J_{0}(\xi \rho)$ is the zeroth-order Bessel function of the first kind, and $N_{0}(\xi \rho)$ is the zeroth-order Neumann function. According to the boundary conditions, i.e., $\bar{\phi}(R)=0$ and $\bar{\phi}(0)=$ finite, we can have $\alpha_{2}=0$ and the condition: $\xi\left(\omega, k_{z}\right)=\beta_{0 n} / R$, where the separation parameter $\xi\left(\omega, k_{z}\right)$ is given by $\xi\left(\omega, k_{z}\right)=k_{z}\left[-1-1 / k_{z}^{2} \lambda_{\kappa e}^{2}+\right.$ $\left.+\omega_{p i}^{2} /\left(\omega-k_{z} u_{i}\right)^{2}+\omega_{p d}^{2} / \omega^{2}\right]^{1 / 2}$ and the coefficient $\alpha_{0 n}(=2.4048,5.5201,8.6537,11.7915)$ are the $n$ th-roots of the zero-order Bessel function of the first kind $J_{0}(\xi \rho)$ at $\rho=R$. The dispersion relation for the space-charge wave in a Lorentzian dusty plasmas is then obtained as

$$
\begin{gathered}
\frac{\omega_{p i}^{2}}{\left(\omega-k_{z} u_{i}\right)^{2}}+\frac{\omega_{p d}^{2}}{\omega^{2}}= \\
=1+\left(\frac{2 \kappa-1}{2 \kappa-3}\right) \frac{1}{k_{z}^{2} \lambda_{D e}^{2}}+\frac{\beta_{0 n}^{2}}{k_{z}^{2} R^{2}},
\end{gathered}
$$

As it is seen in Eq. (10), the instability would occur when the local minimum of the left hand side is greater than $1+[(2 \kappa-1) /(2 \kappa-3)] /$ $/ k^{2} \lambda_{D e}^{2}+\beta_{0 n}^{2} / k_{z}^{2} R^{2}$. Hence, the unstable root $\left(\omega=\omega_{\kappa}+i \gamma_{\kappa}\right)$ is then found to be

$$
\bar{\omega}_{\kappa}\left(\bar{k}_{z}, \kappa, \bar{R}, \beta_{0 n}\right)=\frac{1}{2^{4 / 3}} \frac{\left(\frac{2 \kappa-3}{2 \kappa-1}\right)^{1 / 2} \bar{k}_{z} \bar{R}}{\sqrt{\bar{R}^{2}+\beta_{0 n}^{2}\left(\frac{2 \kappa-3}{2 \kappa-1}\right)}}\left[1+\frac{\left(\frac{2 \kappa-3}{2 \kappa-1}\right) \bar{k}_{z}^{2} \bar{R}^{2}}{\bar{R}^{2}+\beta_{0 n}^{2}\left(\frac{2 \kappa-3}{2 \kappa-1}\right)}\right]^{-1 / 2},
$$

and

$$
\bar{\gamma}_{\kappa}\left(\bar{k}_{z}, \kappa, \bar{R}, \beta_{0 n}\right)=\frac{\sqrt{3}}{2^{4 / 3}} \frac{\left(\frac{2 \kappa-3}{2 \kappa-1}\right)^{1 / 2} \bar{k}_{z} \bar{R}}{\sqrt{\bar{R}^{2}+\beta_{0 n}^{2}\left(\frac{2 \kappa-3}{2 \kappa-1}\right)}}\left[1+\frac{\left(\frac{2 \kappa-3}{2 \kappa-1}\right) \bar{k}_{z}^{2} \bar{R}^{2}}{\bar{R}^{2}+\beta_{0 n}^{2}\left(\frac{2 \kappa-3}{2 \kappa-1}\right)}\right]^{-1 / 2},
$$

where $\bar{\omega}_{\kappa}\left[\equiv\left(\omega_{\kappa} / \omega_{p d}\right)\left(\omega_{p i} / \omega_{p d}\right)^{-1 / 3}\right]$ is the scaled real frequency and $\bar{\gamma}_{\kappa}\left[\equiv\left(\gamma_{\kappa} / \omega_{p d}\right)\left(\omega_{p i} / \omega_{p d}\right)^{-1 / 3}\right]$ is the scaled growth rate, $\bar{k}\left(\equiv k \lambda_{D e}\right)$ is the scaled wave number along the axial $z$-direction, and $\bar{R}\left(\equiv R / \lambda_{D e}\right)$ is the scaled radius of the waveguide. In recent years, the physical characteristics and properties of quantum dusty plasmas have been extensively investigated since the quantum plasma can be found in various nano-materials, nanodevices, and semiconductor plasmas [18-25]. Hence, the investigation of the space-charge wave in a degenerate quantum dusty plasma including the ion streaming will be treated elsewhere.

In order to investigate the influence of nonthermal character and harmonic modes on the spacecharge wave due to ion streaming of ion in a Lorentzian dusty plasma waveguide, we should analyze the dependence of the unstable root $\omega\left(k_{z}, k\right.$, $\left.R, \beta_{0 n}\right)$ on the spectral index $k$ of the Lorentzian plasma and the harmonic root $\alpha_{0 n}$ of the Bessel function. Figure 1 represents the surface plot of the scaled growth rate $\bar{\gamma}_{K}$ of the space-charge wave as a function of the scaled wave number $\bar{k}_{z}$ for various 
values of the spectral index $k$. As shown in this figure, the maximum position of the scaled growth rate $\bar{\gamma}_{\kappa}$ also shifts to the small wave number region as an increase of the spectral index $k$.

Figure 2 represents the surface plot of the derivative of the scaled growth rate $\partial_{\kappa} \bar{\gamma}_{\kappa}\left(\equiv \partial \bar{\gamma}_{\kappa} / \partial \kappa\right)$ of the space-charge wave as a function of the scaled wave number $\bar{k}_{z}$ for various values of the spectral index $k$. As shown in these figures, the maximum position of the scaled growth rate $\bar{\gamma}_{\kappa}$ also shifts to the small wave number region as an increase of the spectral index $k$. In addition, the scaled growth rate $\bar{\gamma}_{k}$ decreases with an increase of the spectral index. Hence, we have found that the growth rates in non-thermal plasmas are always smaller than those in thermal plasmas. From this work, we have shown that the influence of non-thermal character plays a crucial role on the space-charge wave due to the coupling interaction of the streaming ions with non-thermal electrons in a Lorentzian dusty plasma waveguide. These results would provide useful information on the non-thermal effects on the space-charge wave in non-Maxwellian multi-component plasma waveguides.

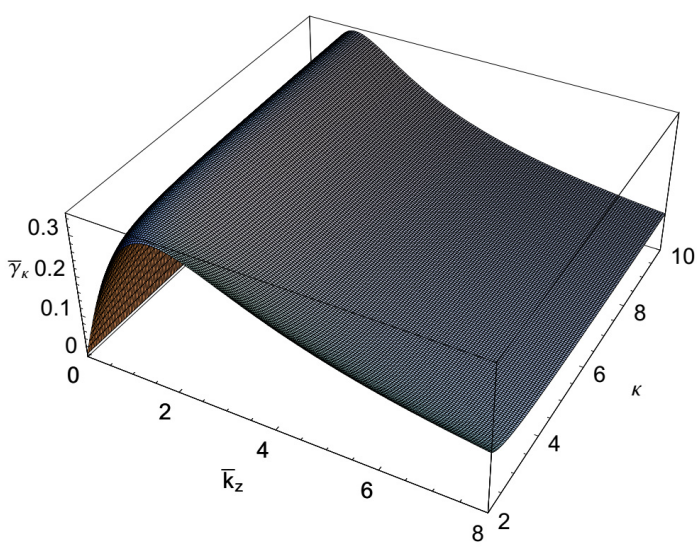

Figure 1 - The surface plot of the scaled growth rate $\bar{\gamma}_{\kappa}$ of the space-charge wave as a function of the scaled wave number $\bar{k}_{z}$ and the spectral index $k$ for the case of the 2 nd-root, i.e., $\alpha_{02}(=2.4048)$ with $\bar{R}=10$

\section{Conclusions}

In conclusion, it is shown that the non-thermal effect constrains the real frequency of the spacecharge wave. Also the scaled growth rate is reducing with an increase of the spectral index. Therefore, it was discovered that the growth rates in non-thermal plasmas are always smaller than that of in thermal plasmas. It was shown that the effect of non-thermal character plays a crucial role on the space-charge wave owing to the coupling interaction of the

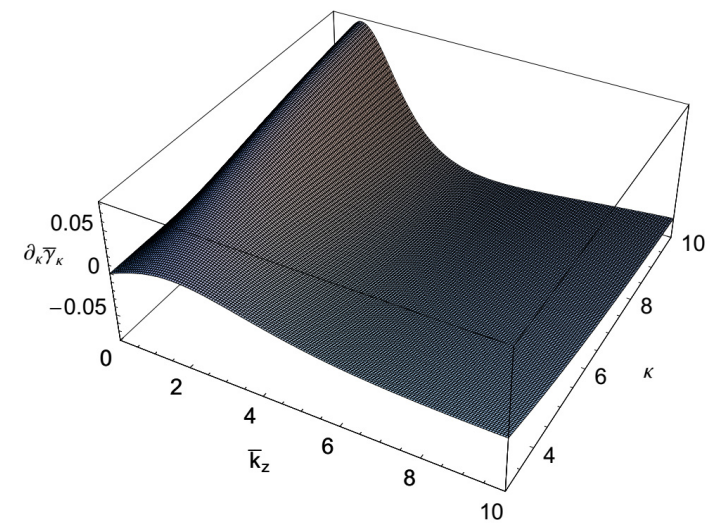

Figure 2 - The surface plot of the scaled growth rate $\partial_{\kappa} \bar{\gamma}_{\kappa}$ of the space-charge wave as a function of the scaled wave number $\bar{k}_{z}$ and the spectral index $\kappa$ for the case of the fourth-root, i.e., $\alpha_{04}(=11.7915)$ with

$$
\bar{R}=10
$$

streaming ions with non-thermal electrons in a Lorentzian dusty plasma waveguide. Useful information on the non-thermal effects on the spacecharge wave in non-Maxwellian multi-component plasma waveguides can be provided by obtained results.

\section{Acknowledgments}

The authors gratefully acknowledge Prof. H. J. Lee for useful comments and discussions. 


\section{References}

1. E. M. Lifshitz, L. P. Pitaevskii. Physical Kinetics. - Amsterdam: Elsevier, 1981.

2. P. A. Sturrock, Plasma Physics. - Cambridge: Cambridge University Press, 1994.

3. K. Nishikawa and M. Wakatani, Plasma Physics. - 3rd ed. - Berlin: Springer-Verlag, 2000.

4. A. F. Alexandrov, L. S. Bogdankevich, A. A. Rukhadze. Principles of Plasma Electrodynamics. - Berlin: Springer, 1984.

5. Yu. M. Aliev, H. Schlüter, A. Shivarova. Guided-Wave-Produced Plasmas - Berlin: Springer, 2000.

6. L. Stenflo, M. Y. Yu. Oscillons at a plasma surface // Phys. Plasmas. - 2003. - Vol. 10. - P. 912.

7. H. J. Lee. Comment on "Kinetic theory of surface waves in plasma jets" Phys. Plasmas 9, 701 (2002)// Phys. Plasmas. - 2005. - Vol. 12. - P. 094701.

8. V. Girka, I. Girka, M. Thumm. Surface Flute Waves in Plasmas. - Cham: Springer, 2014.

9. P. Bliokh, V. Sinitsin, V. Yaroshenko. Dusty and Self-Gravitational Plasma in - Dordrecht : Space Kluwer, 1995.

10. A. Bouchoule. Dusty Plasmas: Physics, Chemistry, and Technological Impact in Plasma Processing Chichester: Wiley, 1999.

11. F. Verheest. Waves in Dusty Space Plasmas. - Dordrecht: Kluwer Academic Publishers, 2000.

12. P. K. Shukla, A. A. Mamun. Introduction to Dusty Plasma Physics. - Bristol: Institute of Physics, 2002.

13. A. Hasegawa, K. Mima, M. Duong-Van, Phys. Plasma Distribution Function in a Superthermal Radiation Field. // Phys. Rev. Lett. - 1985. - Vol. 54. - P. 2608.

14. A. Hasegawa, T. Sato. Space Plasma Physics: Vol. 1, Stationary Processes - Berlin: Springer-Verlag, 1989.

15. N. Rubab, G. Murtaza. Dust-charge fluctuations with non-Maxwellian distribution functions // Phys. Scr. 2006. - Vol. 73. - P. 178.

16. K.-Z. Zhang, J.-K. Xue. Streaming instability in bounded three-component quantum plasmas. // Phys. Plasmas - 2010. - Vol. 17. - P. 032113.

17. N. A. Krall, A. W. Trivelpiece. Principles of Plasma Physics. - New York: McGraw-Hill, 1973.

18. F. B. Baimbetov, Kh. T. Nurekenov, T. S. Ramazanov. Pseudopotential theory of classical non-ideal plasmas // Phys. Lett. A. - 1995. - Vol. 202. - P. 211.

19. T. S. Ramazanov, S. K. Kodanova. Coulomb logarithm of a nonideal plasma // Phys. Plasmas. - 2001. - Vol. 8. - P. 5049.

20. T. S. Ramazanov, K. N. Dzhumagulova. Effective screened potentials of strongly coupled semiclassical plasma. // Phys. Plasmas. - 2002. - Vol. 9. - P. 3758.

21. G. I. Sukhinin, A. V. Fedoseev, T. S. Ramazanov, K. N. Dzhumagulova, R. Zh. Amangaliyeva. Dust particle charge distribution in a stratified glow discharge // J. Phys. D. - 2007. - Vol. 40. - P. 7761.

22. T. S. Ramazanov, K. N. Dzhumagulova, A. N. Jumabekov, M. K. Dosbolayev. Structural properties of dusty plasma in direct current and radio frequency gas discharges. // Phys. Plasmas. - 2008. - Vol. 15. - P. 053704.

23. T. S. Ramazanov, S. K. Kodanova, K. N. Dzhumagulova, N. K. Bastykova. The new method for measuring of dust particles charge in glow discharge plasma. // EPL. - 2011. - Vol. 96. - P. 45004.

24. T. S. Ramazanov, Zh. A. Moldabekov, K. N. Dzhumagulova, M. M. Muratov. Pseudopotentials of the particles interactions in complex plasmas. // Phys. Plasmas. - 2011. - Vol. 18. - P. 103705.

25. Y. A. Ussenov, T. S. Ramazanov, K. N. Dzhumagulova, M. K. Dosbolayev. Application of dust grains and Langmuir probe for plasma diagnostics. // EPL. - 2014. - Vol. 105. - P. 15002. 